\title{
Transportation Ergonomics for Self-Driving Automated Vehicles: Out-dated or Necessary?
}

Easa SM*
Department of Civil Engineering, Ryerson University, Canada

*Corresponding author: Prof. Dr. Said Easa, FCAE, FCSCE, FEIC, FIAAM, Department of Civil Engineering, Ryerson University, Toronto, Canada, Tel: 416-979-5000, ext. 7868, Email: seasa@ryerson.ca

\section{Review Article}

Volume 4 Issue 4

Received Date: June 09, 2020

Published Date: August 10, 2020

DOI: $10.23880 /$ eoij-16000248

\section{Abstract}

While automated vehicles are emerging around the world, many people are unsure what role, if any, ergonomics will play in this new technology. Will ergonomics still be necessary for automated vehicles? This article attempts to answer this question by reviewing key information on two aspects. First, the key elements of ergonomics in human-driven vehicles, including vehicle ergonomics, warehouse ergonomics, training and education, and research and profession, are described. Second, the characteristics of automated vehicles on highways and off highways (e.g. mining and agriculture) are discussed. These two aspects provide the insight needed to determine whether ergonomics are still necessary for automated vehicles and to determine the level of ergonomic requirements. The reader may be surprised to know that self-driving automated vehicles must have drivers, although some special-purpose automated vehicles will be driverless. The results indicate that self-driving automated vehicles have the same ergonomic requirements as human-driven vehicles and that their automated features warrant even more ergonomic research. The results also indicate that ergonomic applications are needed for emerging automated warehouses and off-highway automated vehicles.

Keywords: Transportation; Ergonomics; Automated Vehicles; Standards; Warehouses; Education; Training; Research

\section{Introduction}

Transportation ergonomics is an essential field for human-driven vehicles. The emergence of autonomous (driverless) and connected vehicles, referred to as automated vehicles (AV) in this article, puts into question the necessity of transportation ergonomics for such vehicles. This article attempts to answer this question following a thorough review of the implementation of transportation ergonomics for human-driven vehicles and the characteristics of automated vehicles. This review provides in-depth knowledge of transportation ergonomics and automation that is essential to answer the question posed above.

Automated vehicle technology will reduce human errors and is expected to lead to significant benefits in safety, mobility, and sustainability [1-5]. This technology is emerging worldwide for both passenger and freight vehicles.
AV has already begun to appear on roads across the globe. As the AV market expands, transportation professionals and researchers must address an array of challenges before it becomes a reality. Several government and industry entities have begun to conduct demonstrations and field tests. Centres for testing and validation, education, products, and standards for $\mathrm{AV}$ have been established. Currently, researchers, scientists, and engineers are investing significant resources to develop supporting technologies.

Ergonomics is a scientific discipline that focuses on understanding the interactions between humans and other system elements, where theory, principles, and data, are used to optimize human wellbeing, safety, and system performance. Simply, it is the science of designing systems to fit people. Ergonomics is a multidisciplinary field that draws practitioners and researchers from various specialties, including psychologists, engineers, biologists, 
safety professionals, industrial hygienists, and health care specialists (Fig. 1). The input to the ergonomics science is a blend of principles from psychology (especially human factors), biology, and engineering. Using these principles, ergonomics creates an environment with a low chance of injury. Transportation ergonomics ensures that all aspects of transportation design meet people's needs.

There are numerous types of ergonomics, but the primary types that are applicable to transportation are [6]:

Physical Ergonomics: This is the most common type of ergonomics. This type, sometimes called industrial ergonomics, addresses the response of the human body to the development of physical and physiological workloads. Examples are strain injuries from vibration, force, repetition, and posture.

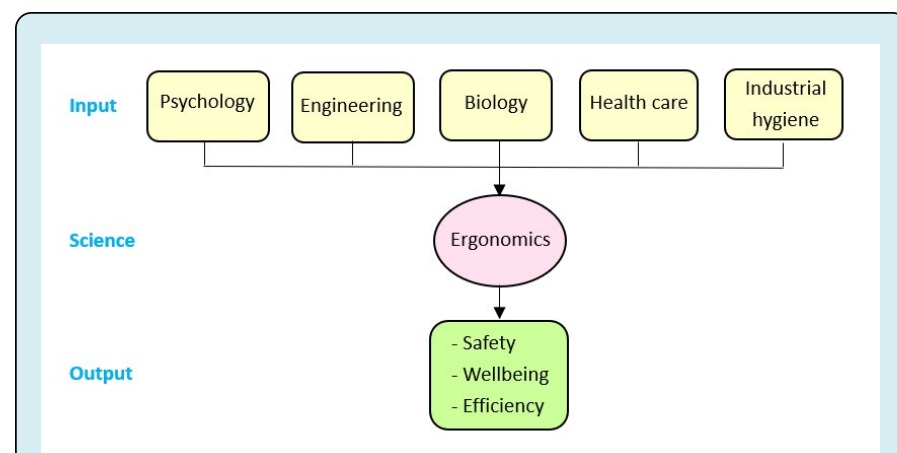

Figure 1: Input and output of ergonomics science.

Cognitive Ergonomics: This type, sometimes called human factors ergonomics, focuses on the study of mental processes (e.g. perception, memory, and reasoning) and the relationship between individuals and other system elements. Examples are mental strain from workload, decision making, and human error.

Organizational Ergonomics: This type aims to optimize the organizational structures, policies, and processes in the work environment. Example include shift work, scheduling, motivation, communication system, and teamwork.

Preventive Ergonomics: This type aims to create awareness among workers about safety in the workplace, the importance of physical and mental health, and the importance of working in comfortable spaces that reduce muscle fatigue.

Environmental Ergonomics: This type focuses on the study of human relations with the physical space, considering such conditions as climate, temperature, pressure, and sound. It determines the most suitable spatial configuration for the development of the proper environment.

\section{Elements of Transportation Ergonomics}

In transportation ergonomics, the preceding types of ergonomics are applied to two areas: vehicles and warehouses. Vehicles include passenger cars, trucks, and buses. Warehouses usually have loading docks to load and unload goods from trucks. They are sometimes designed for the loading and unloading of goods at airports, railways, and seaports. They often have cranes and forklifts for moving goods that are stored as raw materials, packing materials, spare parts, components, or finished goods associated with agriculture, manufacturing, and production [7].

Transportation ergonomics include four basic elements: vehicle ergonomics, warehouse ergonomics, training and education, and research and professional associations, as shown in Fig. 2. Two main categories have been established to promote vehicle ergonomics based on vehicle type: design standards and operational guidelines.

\section{Vehicle Ergonomics}

National and international vehicle ergonomics standards have been developed [8]. The standards provide test procedures for evaluating the factors that affect the performance and comfort of the driver. In particular, the International Organization for Standardization (ISO) covers all aspects of road vehicles, including safety, ergonomics, performance, test methods, environment, and the rollout of innovative technologies [9]. The standards provide benefits for manufacturers and supply chain regulators, health authorities, vehicle owners and drivers, and all road users. In addition to the standards, operational ergonomics guidelines have been established by various organizations. Samples of ergonomics standards and operational guidelines are presented next.

\section{Standards}

The standards for vehicle ergonomics include auditory, tactile, and visual communication between the vehicle and the driver. The following are some standards related to these aspects:

$>$ Road Vehicles - Ergonomics aspects of transportation and control systems:

- Management principles and compliance procedures

- Specifications and test procedures for in-vehicle visual presentation

- Occlusion method to assess visual demand due to the use of the in-vehicle system

$>$ Road Vehicles - Ergonomics and performance aspects of Camera Monitor Systems:

- Requirements and test procedures

- Specifications for in-vehicle auditory presentation

$>$ Road Vehicles - Ergonomics requirements for the driver workplace in line-service buses:

- General description, basic requirements

- Visibility 
- Information devices and control

- Cabin environment

$>$ Ergonomics of the thermal environment - Evaluation of thermal environments in vehicles:

- Principles and methods for the assessment of thermal stress

- Determination of equivalent temperature

- Valuation of thermal comfort using human subjects

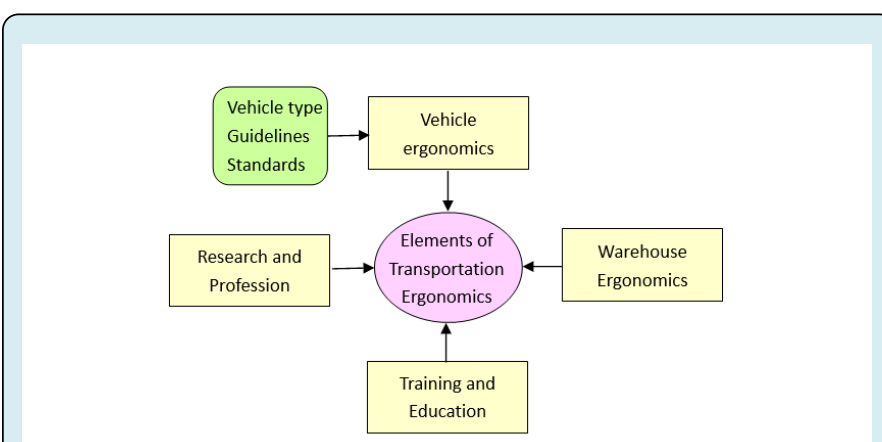

Figure 2: Elements of transportation ergonomics for human-driven vehicles.

\section{Operational Guidelines}

Operational guidelines have been established to promote ergonomics principles when using a vehicle for personal or commercial use, related to: (1) entering and exiting vehicles safely, (2) proper setup of vehicle, (3) manual material handling after driving, (4) using the vehicle as a mobile office, (5) fatigue management, and (6) work relief exercises. Specific operational guidelines are available in the literature [10].

\section{Warehouse Ergonomics}

Truck transportation includes warehouses in which goods are handled by workers who perform numerous tasks that make them prone to injury, especially musculoskeletal disorders (a group of conditions that involve the nerves, muscles, tendons, and supporting structures). The tasks include pushing and pulling carts, lifting and carrying heavy loads, frequent manual material handling tasks, bending and reaching, and twisting of the body. Therefore, implementing ergonomics techniques and best practices in warehouses is necessary to reduce workplace injuries.

A list of best practices related to three ergonomics tasks (lifting and moving, posture-related, and pushing and pulling), based on research by Ware and Fernandez [11] and Tarrant [12], and is presented in Table 1 . These best practices can be applied to all tasks in the warehouse. Lifting and moving items in a warehouse are essential and these best practices ensure that workers are performing these tasks safely. The best practices for awkward postures aim to alleviate risks to muscles and other soft tissues. The best practices for pushing and pulling aim to reduce the forces for pushing and pulling manual material. In addition to these three tasks, workstations should be designed to incorporate ergonomics principles, such as anti-fatigue floor mats and well-designed desk, tables, and chairs. Managers should also ensure that employees have proper training.

\begin{tabular}{|l|l|}
\hline \multicolumn{1}{|c|}{ Task } & \multicolumn{1}{c|}{ Best Practices } \\
\hline \multirow{4}{*}{ Lifting and Moving } & Use lifting aids to reduce awkward postures (twisting and bending). \\
\cline { 2 - 3 } & Use mechanical devices to lift materials to reduce the forces on the body (e.g. pickers). \\
\cline { 2 - 3 } & Use transportation devices to transport materials (e.g. carts, conveyors). \\
\cline { 2 - 3 } & Lift within the "lifting safety zone" (i.e. between elbows and knuckles) \\
\cline { 2 - 3 } & Use proper lifting techniques (i.e. bend hips and knees) \\
\cline { 2 - 3 } & Reduce the frequency of lifting, especially for heavy loads \\
\hline \multirow{5}{*}{ Posture-related } & Work as close to the neutral posture as possible (e.g. straight-down shoulders). \\
\cline { 2 - 3 } & Use work surfaces that have the right height to eliminate bending or reaching. \\
\cline { 2 - 3 } & Use extension poles and adjustable height platforms to avoid reaching. \\
\cline { 2 - 3 } & Make knee pads and stools readily accessible to reduce squatting. \\
\cline { 2 - 3 } & Use a sit/stand stool to reduce the amount of time workers are standing. \\
\hline & Select pushing, rather pulling, as it uses the body's stronger back and leg muscles \\
\cline { 2 - 3 } & Reduce the distance of travel. \\
\cline { 2 - 3 } & Ensure that the cartwheels are well-maintained and work properly. \\
\cline { 2 - 3 } & Perform regular maintenance of manual materials handling carts and casters. \\
\hline
\end{tabular}

Table 1: Best practices for different ergonomics tasks at warehouses. 


\section{Training and Education}

Training for drivers and workers is an important element of transportation ergonomics. Driver training programs focus on ergonomics issues with people who drive frequently or drive long distances. The program typically teaches drivers how their body works and how to prevent injury while driving, lifting, and laptop use in the vehicle as well as stretches that can be done both in and out of the vehicle to prevent discomfort [13]. Worker training programs focus on teaching people the risk factors present in the work environment and how they can mitigate those risks. The training is normally customized to the specific work environment.

For education, many universities offer daytime undergraduate courses and ergonomics certificates as part of continuing education programs. In addition, graduate programs on ergonomics are also available. For example, The University of California, Berkeley offers an online Ergonomics Training Program that has trained graduates and postdocs who have become professors and industry/government leaders. Coursework includes content in engineering, design, physiology, anthropometry, biomechanics, public health, statistics, environmental health sciences, exposure and risk assessment, and root cause analysis [14]. Note that participants must pay fees for ergonomics courses. However, there are free online certificate programs, such as ergonomics in automotive design offered by the Indian Institute of Technology [15]. Many books on ergonomics have been published. The reader is referred to Salvendy [6] and Gkikas [16].

\section{Research and Professional Associations}

Research plays a vital role in advancing the science and practice of ergonomics. Many journals are available for the dissemination of ergonomics research, such as this one. These journals publish developments in areas such as occupational health, musculoskeletal disorders, driver safety, anthropometry, biomechanics, industrial design, kinesiology, physiology, and psychology. There are also research centers that focus on ergonomics.

For example, the Transportation Research Institute at the University of Michigan conducts vehicle ergonomics research that addresses the physical interaction between vehicle occupants and the vehicle interior [17]. The research areas include the effect vehicle interior design factors on driver and passenger posture, position, and comfort. The research aims to develop and upgrade industry practices and models for vehicle design, and to establish criteria for determining levels of occupant accommodation. One interesting development is a new seating accommodation model that predicts the distribution of driver backwardforward positioning based on population stature and key vehicle factors. The model has been applied to develop improved procedures for positioning different-size crash dummies. The research is conducted in cars and trucks driven on-road as well as in several laboratories.

National and international ergonomics associations have been established. Examples include the Association of Canadian Ergonomists, the U.S. Human Factors and Ergonomics Society, the Ergonomics Society of Australia, the Chinese Ergonomics Association, and the International Ergonomics Association. These organizations promote and advance the discovery and exchange of knowledge on human characteristics that are applicable to the design of systems, products, tools, and environments. For example, the U.S. Human Factors and Ergonomics Society has created a National Ergonomics Month (October 2020) to promote wellness and productivity in the workplace. These organizations also hold regular conferences to aid in networking and information exchange. Examples include the Applied Human Factors and Ergonomics Conference (July 2020) and the Applied Ergonomics Conference (August 2020).

\section{Characteristics of Automated Vehicles}

Are ergonomics needed for automated vehicles? Before we can answer this question, we must review some facts about automated vehicles on and off-highways. These facts will provide the rationale and the basis on which to answer the question.

\section{On-Highway Automated Vehicles}

The Society of Automotive Engineers (SAE) International has defined five vehicle automation levels in addition to a driver-only level $[18,19]$. Levels $0-2$ are human driven systems with different levels of automation, while Levels 3-5 are automated driving systems with different levels of control given to the human driver. The six levels are as follows:

\section{Human Driver}

Level 0: Driver only: The human driver performs all driving tasks (steering, brakes, throttle, and power).

Level 1: Assisted automation: A driver assistance system performs either steering or acceleration, while the human driver is expected to carry out the remaining aspects of the dynamic driving task.

Level 2: Partial automation: One or more driver assistance systems perform both steering and acceleration, while the human driver is expected to carry out all remaining aspects of the dynamic driving task. 


\section{Automated Driving System}

Level 3: Conditional automation: An automated driving system performs all aspects of the dynamic driving task (in conditions for which it was designed), but the human driver is expected to respond appropriately to a request to intervene. The driver is not required to monitor the situation in the same way as for the previous levels

Level 4: High automation: An automated driving system performs all aspects of the dynamic driving task and monitor roadway conditions. This level of automation is limited and does not cover every driving scenario.

Level 5: Full automation: An automated driving system performs all aspects of the dynamic driving task under all roadway and environmental conditions. The vehicle is intelligently designed to monitor roadway conditions and act solo, performing all safety-critical driving functions for an entire trip.
Note that Level 3 provides limited self-driving automation under certain traffic or environmental conditions, where the driver cedes full control of all safety-critical functions and relies on the vehicle to watch for any changes in conditions requiring transition to driver control. Then, the driver will be required to resume control of the vehicle after sufficient transition time. Level 5 provides full automation, where the vehicle is intelligently designed to monitor roadway conditions and perform all safety-critical driving functions for an entire trip by itself.

The difference between self-driving and driverless vehicles is worth noting. Levels 3 and 4 automation must have a driver, and therefore these levels refer to self-driving automated vehicles (the driver must be present). On the other hand, Level 5 does not need a driver and this level may be referred to as driverless automated vehicles.
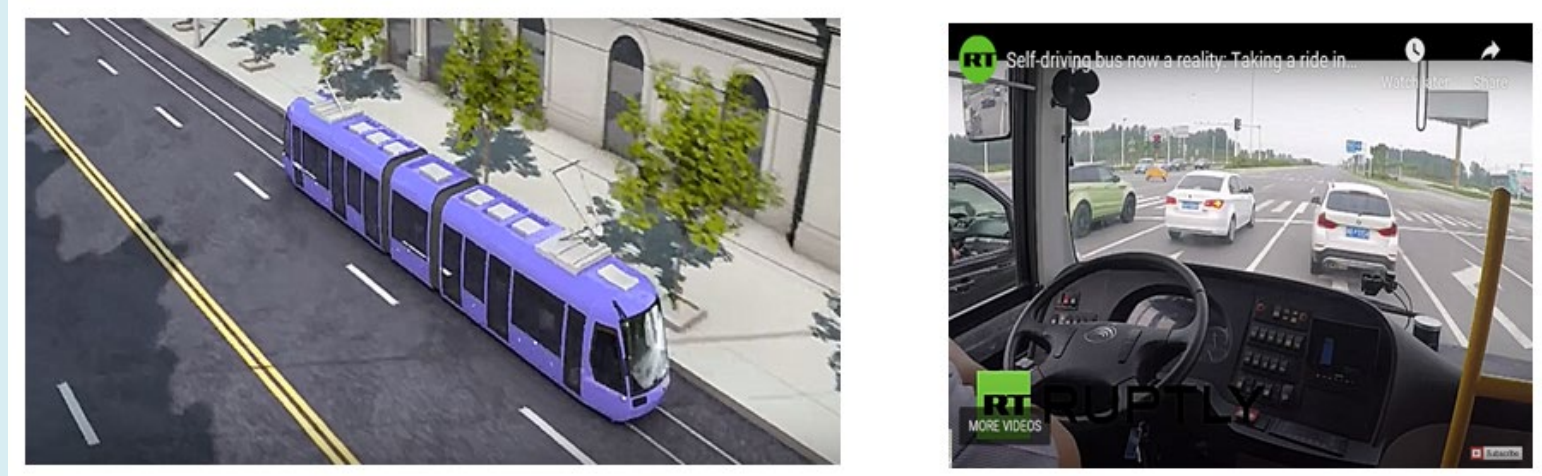

Figure 3: Self-driving automated bus: (a) Bus on virtual rail (developed by CRRC Zhuzhou Institute Co) and (b) safety driver on automated bus (developed by Zhengzhou Yutong Group Co).

For automated buses, most systems that have been implemented in China, Singapore, and elsewhere use a 'safety' driver who takes control of the bus only in emergency situations [20]. A driverless fully automated bus on a virtual rail that became operational last year in China, is shown in Figure 3. Automated parking technology has also been developed and implemented. This technology supports both parking and driving out regardless of whether the driver is in the vehicle or not. After the driver disembarks the vehicle and presses the button on the Smart Key, the vehicle begins to park itself. This mode supports vertical and parallel parking alike [21].

\section{Off-Highway Automated Vehicles}

Automated equipment is growing in popularity in numerous off-highway application areas, such as agriculture, mining, and construction. As these industries move toward automated operation, changes in off-highway vehicle/ equipment design are needed to ensure the equipment is better used, safer, and more productive.

Straka [22] highlighted several applications of advanced technology to address the challenges of operator ergonomics:

- Change the ergonomics within the cab by increasing the number of screens within the cab interior to help the operator's better monitor the status of the equipment. Since different operators work in these cabs, the screens need to be positioned to account for the height differences of each operator.

- Use position control technology (e.g. torque hinges and display arms) to: (a) adjust screens to suite operator preferences to prevent strain, (b) adjust for glare, and (c) maximize operator visibility. The same technology can also be used in smartphone and tablet mounts that may be used in the cab.

- $\quad$ Outside of the cab, address the variety of access points of the off-highway equipment, such as tool cabinets and 
hoods covering engines which should be tightly secured and easily accessible.

- Improve operator cab air quality by refining the heating, ventilating, and air conditioning systems in the vehicles (e.g. sealing the cabs to better control cool air leakage and keep the dust out).

- Improve operator safety and visibility by adding a small camera on the side of the mirror or on the door that gives the operator a broader view of the surrounding area. This would represent a step toward fully automated driving.

\section{Are Ergonomics Needed for AV?}

It is clear from the $\mathrm{AV}$ characteristics and the review of the ergonomics elements for human-driven vehicles that, in general, the application of ergonomics will still be needed for AV vehicles. First, passenger cars with Level 3 automation will have both human-driver and automated modes. Thus, the ergonomics needs of the driver still exist. Even for Level 4 full automation, the driver will still be present in the car and the application of most ergonomics principles is still necessary. Second, for automated trucks, most ergonomics design standards and operational guidelines will still be applicable. Third, since most automated buses will have safety drivers, most ergonomic requirements will not change. Off-highway automated vehicles also require the application of ergonomics principles to ensure the wellbeing of vehicle operators.

It should be noted, however, that some special types of highway automated vehicles that require no driver involvement for operation would require no ergonomics implementation. For example, driverless automated vehicles have been deployed to deliver prescriptions for CVS Pharmacy across three zip codes in Houston, Texas [23]. Prescription and non-prescription orders can be placed by customers in the pilot area on the CVS website. By selecting the automated delivery option, one of Nuro's Toyota Priuses will deliver the order to the curbside at the customers address within three hours. Customers then confirm their identity and unlock the delivery when the automated vehicle arrives.

Researchers are beginning to address the human factors and ergonomics issues that are associated with automated vehicles (Figure 4). For example, researchers at the University of Michigan are developing several experimental protocols to address some issues related to automation (e.g. inattentive driving and trust) and efficient instruction methods to improve driver interaction with automated vehicles. Other critical issues include safety of vulnerable road users and evaluating the design and usability of vehicle controls, displays and other AV information systems [24-26].
What is interesting is that many human factor experts are of the opinion that $\mathrm{AV}$ would require more ergonomics research. Kyriakidis et al. [27] conducted interviews with 12 expert researchers in the field of human factors in automated driving to identify different perspectives regarding relevant challenges in AV development. The experts indicated that: (1) the automated vehicle should ensure safety while changing automated to human-driver mode and should inform its driver of its capabilities and operational status, (2) research should address the HF-related interactions between AV and vulnerable road users, such as pedestrians and cyclists, and (3) the need to modify driver-training programs to ensure that humans are capable of using automated vehicles. While reflecting on the interviews, the authors also indicated that the rapid developments in AV technology are not in agreement with the consensus among the interviewees which was consistent with a long history of HF research. The authors' perspective is therefore expected to be instrumental in $\mathrm{AV}$ development.

A significant human-factors issue in self-driving automated vehicles (Levels 3 and 4) is the shift from automated to human-driver mode. When the driver is alerted by the vehicle-user interface regarding the need to shift, the driver must have sufficient time to take over the manual control of the vehicle. This time depends on the driver's level of situation awareness and the complexity of the situation. In addition, higher levels of automation foster complacency, where the driver may engage in non-driving tasks. Those drivers would need substantially longer time to regain situation awareness and reenter the driving control mode. Therefore, the automated system must be designed to accommodate the majority of the drivers (e.g. 99-percentile value), similar to the case of perception-reaction time in highway geometric design [28]. The modeling of driver response should be based on uncertainty analysis [29-31], where a very high reliability level should be used in this case. For more details on this and other human factors issues, the reader is referred to the excellent article by Grugle [32].

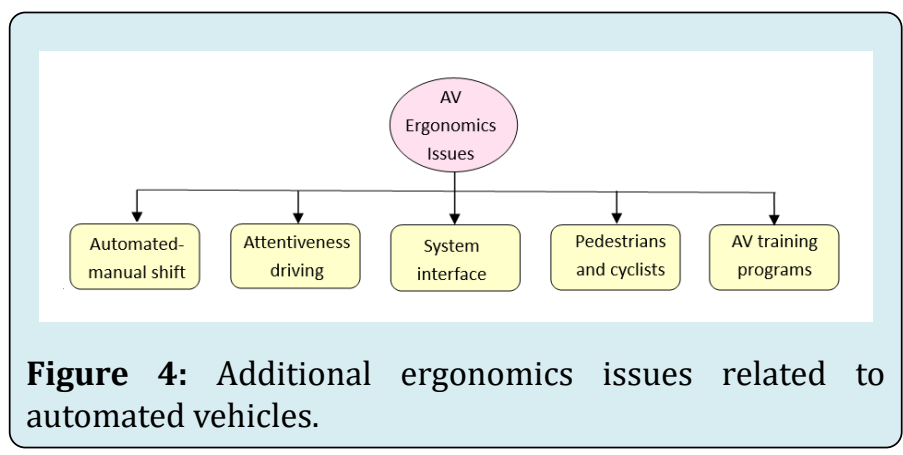

Another question emerges: Are more ergonomics needed for warehouses? The answer is Yes. With the 
growing trend of vehicle automation, some warehouses are becoming completely automated, where pallets and products automatically move on a system of conveyors, cranes, and automated storage. Such systems are commonly used in refrigerated warehouses and where land is expensive since automated storage systems can efficiently use vertical storage. To function efficiently, a warehouse should be properly slotted to improve its inventory rotation requirements, labour cost, and productivity [7]. Clearly, like off-highway automated vehicles, automation of warehouses creates new challenges in applying ergonomics to ensure the safety and wellbeing of workers.

\section{Conclusion}

This article has presented a review of the elements of ergonomics applications for human-driven vehicles and the characteristics of emerging automated vehicles to answer the question: Will ergonomics still be necessary for automated vehicles? The review revealed that most self-driving automated vehicles not only need the same ergonomics requirements currently used in conventional vehicles, but also that their automated features warrant more ergonomics research. The new research areas are related to the shift from automated to human-driver mode, attentive driving, interactions with pedestrians and cyclists, and new AV training programs.

Research has established that short-term discomfort is related to long-term injury and impairment later in life. Early intervention through the implementation of ergonomics is therefore essential. This highlights the importance of establishing specialized research centers and academic programs that focus on the impact of automation on human factors and ergonomics.

Warehouse automation and off-highway vehicle automation are also increasing. These new automated systems require new types of ergonomics applications to ensure the safety and wellbeing of operators. The emerging automated systems for vehicles and warehouses provide numerous challenges and opportunities to develop innovative ergonomics applications. Bus stop ergonomics, which have been somewhat lacking in practice, were not addressed in this article, but they will become important as smart technologies are implemented at bus stops and terminals. With the opportunities for automated vehicles on and offhighway on the rise, organizations optimistic about the future role of ergonomics in automated systems.

\section{Acknowledgement}

This research is supported by a Discovery Grant from the Natural Sciences and Engineering Research Council of
Canada.

\section{References}

1. Narla S, Stowell H (2019) Connected and autonomous vehicles. Institute of Transportation Engineers Journal March Issue.

2. Elliott D, Keen W, Miao L (2019) Recent advances in connected and automated vehicles. Journal of Traffic and Transportation Engineering 6(2): 109-131.

3. Shladover S (2017) Connected \& automated vehicle systems: Introduction and review. Intelligent Transportation Systems Journal 22(3): 190-200.

4. NCHRP (2017) Connected \& autonomous vehicles and transportation infrastructure readiness. Project, National Cooperative Highway Research Program, pp: 20-24.

5. Stanton N (2019) Advances in human factors of transportation. Proc., International Conference on Applied Human Factors and Ergonomics, Washington DC, USA.

6. Salvendy G (2012) Handbook of human factors and ergonomics, Wiley, New York, USA.

7. Wikipedia (2020) Warehouses.

8. ANSI (2020) Vehicle ergonomics standards, American National Standards Institute, New York, United States. International Organization for Standardization.

9. ISO (2020) Standards. International Organization for Standardization.

10. Antich M (2017) Improving truck ergonomics for driver safety.

11. Ware BF, Fernandez JE (2014) Warehouse ergonomics/ tips and techniques to decrease injury risk Environmental, Health, and Safety.

12. Tarrant S (2019) Ergonomics best practices in the manufacturing industry.

13. Anderson Ergonomics Consulting (2020) Industrial ergonomics training.

14. Center for Occupational and Environmental Health (2020) CE ergonomics program. University of California, Berkeley.

15. Indian Institute of Technology (2020) Ergonomics in automotive design. In: Gkikas N, (Ed.), Automotive 
ergonomics driver-vehicle interaction, CRC Press.

16. Gkikas N (2017) Automotive ergonomics driver-vehicle interaction, CRC Press, Boca Raton, Florida, USA.

17. Transportation Research Institute (2020) Vehicle ergonomics, University of Michigan, USA.

18. SAE International (2014) Taxonomy and Definitions for Terms Related to On-Road Motor Vehicle Automated Driving Systems.

19. SAE International (2016) Taxonomy and Definitions for Terms Related to Driving Automation Systems for OnRoad Motor Vehicles.

20. Easa SM (2020) Smart Cities require smart public transportation systems. Keynote Presentation, International Conference on Smart City Engineering and Public Transportation, Guangzhou, China (online).

21. Diachuk M, Easa SM, Bannis J (2020) Path and control planning for autonomous vehicles in restricted space and low speed. Infrastructures Journal 5(44): 1-25.

22. Straka B (2020) New challenges in off-highway equipment design.

23. Muir J (2020) Nuro delivers prescriptions for CVS. Autonomous Vehicle International.

24. Stanciu S, Eby DW, Molnar LJ, St. Louis RM, Zanier N, et al. (2018) Pedestrians/bicyclists and autonomous vehicles: How will they communicate? Transportation Research Record 2672(22): 58-66.
25. Stachowski S, Gaynier R, LeBlanc DJ (2019) An assessment method for automotive intrusion detection system performance. DOT HS 812708 National Highway Traffic Safety Administration.

26. Molnar LJ, Pradhan AK, Eby DW, Ryan L, St. Louis RM, etal (2017) Age-Related Differences in Driver Behavior Associated with Automated Vehicles and the Transfer of Control between Automated and Manual Control: A Simulator Evaluation. Report No. UMTRI-2017-4.

27. Kyriakidis M, de Winter J C, Stanton N, Bellet T, van Arem B, et al. (2020) A human factors perspective on automated driving. Theoretical Issues in Ergonomics Science 20(3): 223-249.

28. AASHTO (2018) A Policy on Geometric Design of Highway and Streets, American Association of State Highway and Transportation Officials.

29. Easa SM, Diachuk M (2020) Optimal speed plan for overtaking of autonomous vehicles on two-lane highways. J Infrastructures 5(5): 44.

30. Greto K, Easa SM (2019) Reliability-based design of truck escape ramps. Canadian Journal of Civil Engineering 47(4): 395-404.

31. Easa SM, Cheng J (2013) Reliability analysis of minimum pedestrian green interval for traffic signals. Journal of Transportation Engineering 139(7): 651-659.

32. Grugle N (2019) Human factors in autonomous vehicles. American Bar Association. 